\title{
Superconductivity of Niobium Thin Films in the $\mathrm{BiOCl} / \mathrm{Nb}$ Heterostructures
}

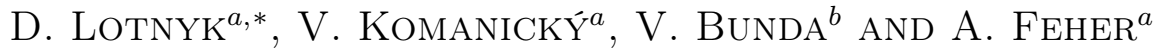 \\ ${ }^{a}$ Institute of Physics, Faculty of Science, P.J. Šafárik University, Park Angelinum 9, 04154 Košice, Slovakia \\ ${ }^{b}$ Transcarpathian Institute of Arts, Voloshiv Str. 37, 88000 Uzhgorod, Ukraine
}

\begin{abstract}
In the current paper, electrical transport properties of $25 \mathrm{~nm}$ thick $\mathrm{Nb}$ films sputtered on the photosensitive semiconductor $\mathrm{BiOCl}$ were investigated in the temperature range $7.5 \mathrm{~K} \leq T \leq 8.5 \mathrm{~K}$. The influence of green $(532 \mathrm{~nm})$ and red $(640 \mathrm{~nm})$ laser excitations on resistive superconducting transitions of the niobium thin films on a silicon glass and $\mathrm{BiOCl}$ single crystal substrates were studied. The temperature dependences of the resistivity for $\mathrm{Nb}$ are in good agreement with the McMillan model which indicates the strong influence of the inverse proximity effect induced by the interface. The increased influence of the $\mathrm{BiOCl} / \mathrm{Nb}$ interface under laser excitation corresponds to the raising of the ratio of the density of normal to superconductivity carriers in the $T \rightarrow 0$ limit and this observation is in agreement with the photoconductivity study of $\mathrm{BiOCl}$ single crystals.
\end{abstract}

DOI: 10.12693/APhysPolA.131.1030

PACS/topics: 74.25.F-, 68.35.bg, 74.45.+c

\section{Introduction}

In the past decade, attention was paid to the lightinduced effect in superconductors [1-3]. Under laser excitation, it is possible to manipulate the surface density of state, to change superconducting properties. Another possibility to change the local density of states arises from proximity induced effects [4]. One can manipulate the superconducting density of states at the interface using photosensitive semiconductor/superconductor heterostructures. Bismuth oxyhalides $\mathrm{BiOX}(\mathrm{X}=\mathrm{F}$, $\mathrm{Cl}, \mathrm{Br}$ and I) photosensitive semiconductors that have attracted intensive attention due to their characteristic photoelectric properties and possible technological applications $[5,6]$. Due to their layered structure and facile fabrication by exfoliation [7], single crystals with thicknesses less than $1 \mu \mathrm{m}$ could be used as a substrate for semiconductor/superconductor heterostructures.

The electrical transport properties of thin $\mathrm{Nb}$ films, with nominal thicknesses of $25 \mathrm{~nm}$, supported on Si glass or $\mathrm{BiOCl}$ single crystal substrates were investigated in the temperature range $7.5 \mathrm{~K} \leq T \leq 8.5 \mathrm{~K}$ with and without laser excitation. The temperature dependences of resistivity for $\mathrm{Nb}$ on $\mathrm{Si}$ glass overlapping with each other indicating an excellent stability of interface. The resistive curves at the vicinity of superconducting transition for $\mathrm{Nb}$ on $\mathrm{BiOCl}$ single crystal are in good agreement with the McMillan model [8], which indicates the strong influence of inverse proximity effect induced by the interface. The calculated depth of the interface is approximately $0.85 \mathrm{~nm}$. The increased influence of the interface under the laser excitation corresponds to the raising in

\footnotetext{
* corresponding author; e-mail dmytro.lotnyk@student .upjs.sk
}

$N_{N}(0) / N_{S}(0)$ ratio $\left(N_{N, S}(0)\right.$ are the density of states in normal $(\mathrm{N})$ and superconducting $(\mathrm{S})$ layers at $T=0)$. It is in agreement with the photoconductivity spectra of $\mathrm{BiOCl}$ single crystals obtained in [9].

\section{Experimental details}

In this work, a magnetron sputtering technique was used to generate $\mathrm{Nb}$ films with nominal thicknesses of $25 \mathrm{~nm}$. Sputtering conditions were: chamber pressure $3.7 \times 10^{-10} \mathrm{~Pa}$, argon pressure $3 \times 10^{-5} \mathrm{~Pa}$, DC target power $270 \mathrm{~W}$, substrate temperature $22^{\circ} \mathrm{C}$. The thickness of the sputtered films was controlled by quartz crystal microbalance device. The films were simultaneously deposited on commercially purchased Si glass slides $20 \mathrm{~mm} \times 20 \mathrm{~mm} \times 0.5 \mathrm{~mm}$ and $\mathrm{BiOCl}$ single crystal $10 \mathrm{~mm} \times 5 \mathrm{~mm} \times 1 \mu \mathrm{m}$ obtained by exfoliation method [7]. The high-quality $\mathrm{BiOCl}$ single crystal that served as the source of the thin films was synthesized using gas transfer methods briefly described in [9].

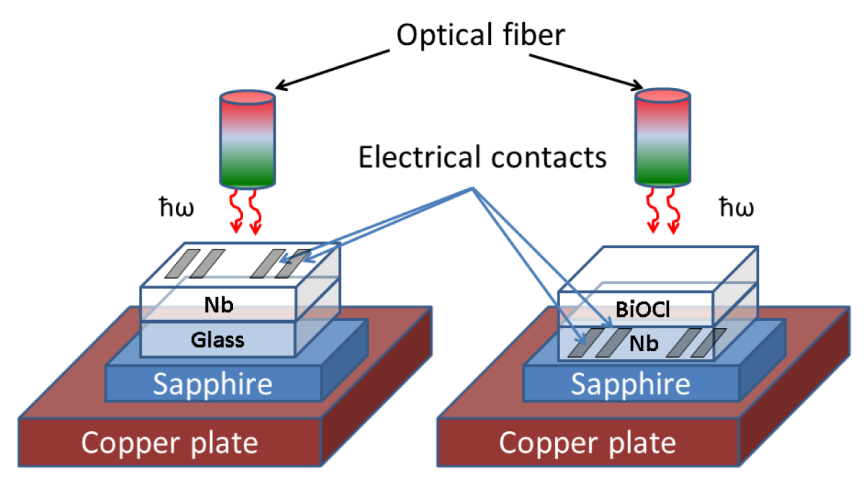

(a)

(b)

Fig. 1. Schematic representation of electric transport measurements. (a) $25 \mathrm{~nm} \mathrm{Nb}$ sputtered on the Si glass and (b) $25 \mathrm{~nm} \mathrm{Nb}$ sputtered on the BiOCl. 
The electrical resistivity of the $\mathrm{Nb}$ thin films was obtained by the four-point probe measurements of a direct current using Keithley 6221 current source and Keithley $2182 \mathrm{~A}$ nanovoltmeter. Temperature control was performed using the commercial Physical Property Measurement System (PPMS). Temperature dependences were obtained in both cooling and heating modes at two temperature rates $0.1 \mathrm{~K} / \mathrm{min}$ and $0.3 \mathrm{~K} / \mathrm{min}$. Thermal contact was established by installing sample on a massive $\mathrm{Cu}$ plate glued with the GE-Varnish (see scheme in Fig. 1). Electric contacts were made using the silver conducting paste with annealing at $80^{\circ} \mathrm{C}$ during $10 \mathrm{~min}$. The thin sapphire plate was used to avoid short circuit or current leaking. Laser excitation with the power $15 \mathrm{~mW} / \mathrm{cm}^{2}$ was applied on either $\mathrm{Nb}$ (Fig. 1a) or the photosensitive semiconductor $\mathrm{BiOCl}$ (Fig. 1b). Laser excitations with $532 \mathrm{~nm}(2.33 \mathrm{eV})$ and $640 \mathrm{~nm}(1.94 \mathrm{eV})$ were obtained by two CNI laser devices with the maximum power $300 \mathrm{~mW}$ and $200 \mathrm{~mW}$, respectively. Output laser power was measured using thermal power sensor (Ophir, 3A-P). Resistive curves were measured at the current $1 \mu \mathrm{A}$.

\section{Results and discussion}

The experimental results of the superconducting transition for the $\mathrm{Nb}$ films under various mounting and irradiation conditions are given in Fig. 2. It is noteworthy that the data for the transitions were acquired with warming and cooling protocols at two different rates, and the results for a specific mounting/irradiation conditions were independent of the various measuring conditions. This observation indicates that a high-quality thermal contact exists between the sample and the cold plate of the PPMS, and consequently, the laser excitation does not significantly perturb the temperature of the sample. Only the curves measured at temperature rate $0.1 \mathrm{~K} / \mathrm{min}$ in cooling mode are plotted in Fig. 2.

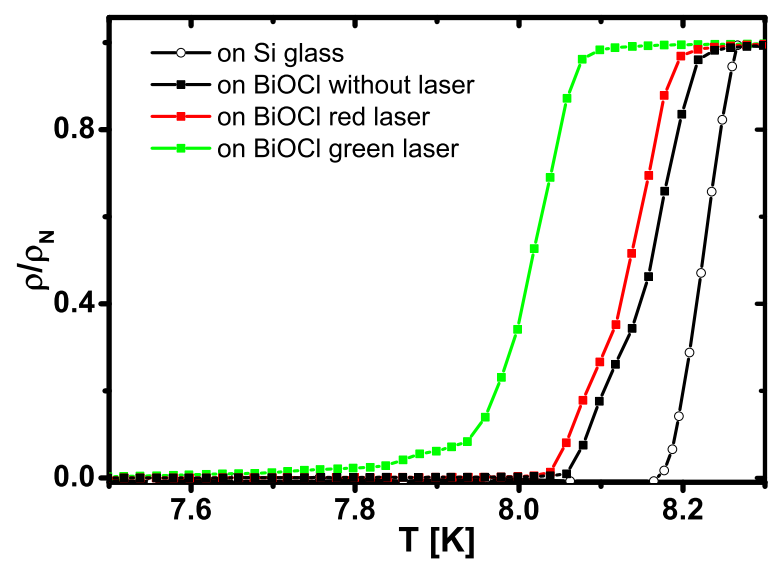

Fig. 2. Temperature dependences of normalized resistivity of $25 \mathrm{~nm} \mathrm{Nb}$ thin films on Si glass (circles); on $\mathrm{BiOCl}$ without laser excitation (black squares), under green (green squares) and red (red squares) laser excitation.
Critical temperatures were identified by the maximum value of $\mathrm{d} \rho / \mathrm{d} T$ curves (Fig. 3), and the results are tabulated in Table I. The width of superconducting transitions was identified in the temperature range from deviation from normal state behavior till the state $\rho \rightarrow 0$ (Fig. 2). The thin film sputtered on Si glass has a critical temperature $8.2 \mathrm{~K}$ along with the width of superconducting transition $\Delta T_{c} \approx 0.2 \mathrm{~K}$ and the residual resistance ratio $(\mathrm{RRR}) \approx 4.3$ indicates a high quality of the thin film. All measured curves, both without and with laser excitation, perfectly match each other, and this result is a consequence of an interface with excellent stability.

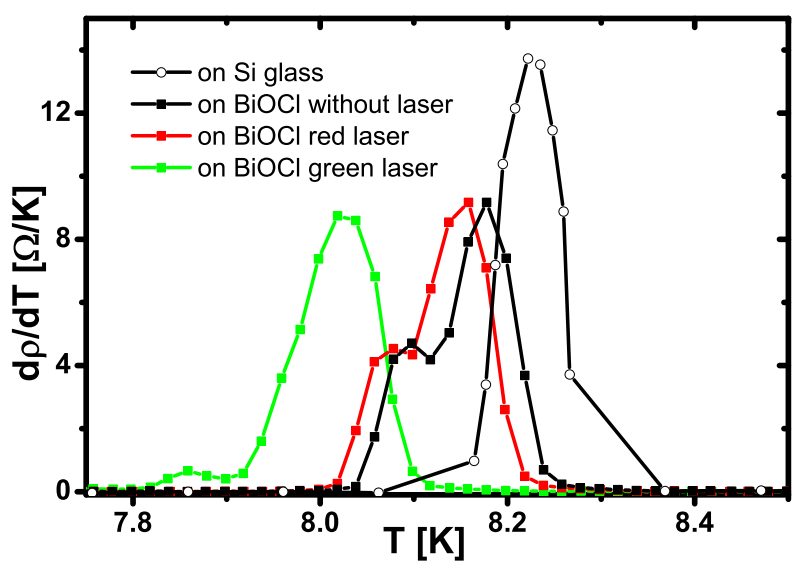

Fig. 3. Temperature dependences of $\mathrm{d} \rho / \mathrm{d} T$ for $25 \mathrm{~nm}$ $\mathrm{Nb}$ thin films on $\mathrm{Si}$ glass (circles); on $\mathrm{BiOCl}$ without laser excitation (black squares), under green (green squares) and red (red squares) laser excitation.

The superconducting transitions of $\mathrm{Nb}$ on the $\mathrm{BiOCl}$ substrate shift to the lower temperatures with increase of the laser excitation energy. The residual resistance ratio measured without laser excitation 3.8, which is slightly lower compared to the Si glass substrate. The higher width of the superconducting transition $\Delta T_{c}$ and the presence of the second, low-temperature peak $\mathrm{d} \rho / \mathrm{d} T$ curves could indicate the phase separation in $\mathrm{Nb}$ thin films. With and without irradiation, the RRR for $\mathrm{Nb}$ on $\mathrm{BiOCl}$ is the same, so the defect concentration is constant during all measurements. On the other hand, the width of the superconducting transition $\Delta T_{c}$ usually indicates the impureness of the sample and this parameter increased sufficiently under laser excitation.

TABLE I

Critical temperatures $T_{c}$ and the width of superconducting transitions $\Delta T_{c}$ of $25 \mathrm{~nm}$ thin films on Si glass and on the $\mathrm{BiOCl}$ substrates.

\begin{tabular}{c|c|c|c|c}
\hline \hline \multirow{2}{*}{ Conditions } & \multicolumn{2}{|c|}{$T_{c}[\mathrm{~K}]$} & \multicolumn{2}{c}{$\Delta T_{c}[\mathrm{~K}]$} \\
\cline { 2 - 5 } & $\mathrm{Si}$ glass & $\mathrm{BiOCl}$ & Si glass & $\mathrm{BiOCl}$ \\
\hline without laser & 8.22 & 8.17 & 0.2 & 0.24 \\
\hline red light & 8.22 & 8.15 & 0.2 & 0.28 \\
\hline green light & 8.22 & 8.02 & 0.2 & 0.35
\end{tabular}


Such a behavior could be explained regarding inverse proximity effect [10]. Despite the good quality of thin films, which indicates in $\mathrm{RRR} \approx 4$, the pure $\mathrm{Nb}$ single crystals have RRR up to 2600 [11]. Consequently, the approximation in dirty limit could be used. According to Ashcroft [12], the electron mean free path was estimated as $l=\frac{\left(r_{s} / a_{0}\right)^{2}}{\rho_{\mu}} \times 92 \AA$, where $\rho_{\mu}$ is a sheet resistance in $\mu \Omega \mathrm{cm}$ and ratio $r_{s} / a_{0}$ for $\mathrm{Nb}$ equals 3.07 . The experimental value of sheet resistance is $\rho_{\mu}=3.09 \mu \Omega \mathrm{cm}$, which leads to the $l \approx 28 \mathrm{~nm}$. Since the sample thickness $d$ is less than the electron mean free path $l$, the former was used to evaluate the coherence length in the dirty limit [12] as $\xi=0.852 \sqrt{\xi_{0} d}$. Assuming $\xi_{0}=38 \mathrm{~nm}$ for bulk $\mathrm{Nb}$ [10], then this analysis yields $\xi=26 \mathrm{~nm}$. This value is very close to the thickness of our $\mathrm{Nb}$ film $d=25 \mathrm{~nm}$ and places our study in the two-dimensional limit. According to the McMillan model [8], the inverse proximity effect in a planar thin film geometry is an appropriate description of our experimental conditions. Consequently, the critical temperature $T_{c}$ is suppressed by the normal state interface with the thickness $d_{N}$ described by the equation

$$
T_{c}=T_{c 0}\left(\frac{3.56 T_{D}}{T_{c 0} \pi}\right)^{-\alpha / d},
$$

where $T_{D}=277 \mathrm{~K}$ is the $\mathrm{Nb}$ Debye temperature, $T_{c 0}=9.22 \mathrm{~K}$ is the $\mathrm{Nb}$ bulk critical temperature [10] and $\alpha=d_{N} N_{N}(0) / N_{S}(0)$. Considering a $N_{N}(0) / N_{S}(0)$ equals to one without laser excitation, the calculated value of $d_{N}$ was estimated as $0.85 \mathrm{~nm}$. Under the laser excitation, the only parameter in Eq. (1) being changed is $\alpha$. Consequently, increase of the $N_{N}(0) / N_{S}(0)$ ratio leads to the suppression of the superconductivity. Changes for $N_{N}(0) / N_{S}(0)$ ratio are 1.02 under the red light excitation and 1.15 under the green light excitation. Such behavior is in good agreement with photoconductivity spectra obtained in [9] for $\mathrm{BiOCl}$ single crystals.

\section{Conclusions}

The electrical transport properties of $25 \mathrm{~nm} \mathrm{Nb}$ thin films were investigated in a temperature range $7.5 \mathrm{~K} \leq T \leq 8.5 \mathrm{~K}$ on $\mathrm{Si}$ glass and $\mathrm{BiOCl}$ substrates with and without laser excitation. Resistive curves with and without laser excitation for $\mathrm{Nb}$ on $\mathrm{Si}$ glass matched with each other which indicates a good stability of interface and parameters in Eq. (1). The temperature dependences of resistivity of $\mathrm{Nb}$ on $\mathrm{BiOCl}$ single crystal are in good agreement with the McMillan model which indicates the strong influence of inverse proximity effect induced by the interface. The calculated depth of the normal state interface is $d_{N} \approx 0.85 \mathrm{~nm}$. The increased influence of the interface under the laser excitation corresponds to the enlarging in $N_{N}(0) / N_{S}(0)$ ratio which is in agreement with the photoconductivity study of $\mathrm{BiOCl}$ single crystals.

\section{Acknowledgments}

This work was supported by the ERDF EU (European Union European regional development fond) grant, under the contract No. ITMS 26220120005, ITMS 26220220186, APVV 0605-14 and VEGA 1-0409-15. We thank Professor M.W. Meisel for the fruitful discussions and comments that improved the manuscript.

\section{References}

[1] D. Fausti, R.I. Tobey, N. Dean, S. Kaiser, A. Dienst, M.C. Hoffmann, S. Pyon, T. Takayama, H. Takagi, A. Cavalleri, Science 331, 189 (2011).

[2] M. Mitrano, A. Cantaluppi, D. Nicoletti, S. Kaiser, A. Perucchi, S. Lupi, P. Di Pietro, D. Pontiroli, M. Ricco, S.R. Clark, D. Jaksch, A. Cavalleri, Nature 530, 461 (2016).

[3] M. Suda, R. Kato, H.M. Yamamoto, Science $\mathbf{3 4 7}$ 743 (2015).

[4] P.G. de Gennes, Rev. Mod. Phys. 36, 225 (1964).

[5] S.J. Wu, C. Wang, Y.F. Cui, T.M. Wang, B.B. Huang, X.Y. Zhang, X.Y. Qin, P. Brault, Mater. Lett. 64, 115 (2010).

[6] M.A. Gondal, X.F. Chang, M.A. Ali, Z.H. Yamani, Q. Zhou, G.B. Ji, Appl. Catal. A Gen. 397, 192 (2011).

[7] A.K. Geim, K.S. Novoselov, Nature Mater. 6, 183 (2007).

[8] W.L. McMillan, Phys. Rev. 175, 537 (1968).

[9] V. Bunda, S. Bunda, in: Superconductors - Its Properties, Technology, and Applications, Ed. Yu. Grigorashvili, InTech, Rijeka 2012, p. 323.

[10] C. Delacour, L. Ortega, M. Faucher, T. Crozes, T. Fournier, B. Pannetier, V. Bouchiat, Phys. Rev. B 83, 144504 (2011).

[11] E.U. Malang, C.S. Oglesby, T. Siegrist, E. Bucher, Physica B Condens. Matter 204, 363 (1995).

[12] N.W. Ashcroft, N.D. Mermin, Solid State Physics, Holt, Rinehart and Winston, New York 1976. 